\title{
Design and Implementation of Low Voltage Three Phase Motor Speed Control and Visual Monitoring
}

\author{
Sunomo, Herlambang Sigit, Andik Asmara \\ Faculty of Engineering, Universitas Negeri Yogyakarta \\ Yogyakarta, Indonesia
}

\begin{abstract}
This paper focused on the creation of three phase low voltage motor control under 50 volt for Power Electronic Lab Course of vocational education. With HEF 4752 PWM IC and microcontroller based system, motor operated in three category; low, medium and high speed. Under three phase 32 volt input, the motor runs at RPM 573 in $\mathbf{1 1 . 5 0}$ volt for low speed, RPM 1104 in 20.50 volt for medium speed, and RPM 2022 in 26 volt for high speed.. The step of frequency and voltage output is not linear with the value of resistor control setting. Motor can be connected in star or delta unloaded mode. In delta connection with resistive virtual ground for the oscilloscope, the voltage shapes are loss of sinusoidal pattern.
\end{abstract}

Keywords: motor control, hef 4752 , three phase, power electronic laboratory course

\section{INTRODUCTION}

Currently, variable frequency drive for three phase motor has been familiar in various industry. The technology principle inside must be introduced to the student of vocational education visually in the save manner; ie, electric shock and the electronic instrument measuring system safety. So, this paper proposed low voltage three phase experiment modul that works bellow 50 volt, ie, 32 volt per phase.

Many electronic circuit of Variabel voltage and variable frequency (VVVF) drive with PWM mode now have been available in the market. The one of them is HEF 4752 from Philips Semiconductor. HEF 4752 PWM motor control has been used booth by Patil-Kurkute (2006) and Bellil-Meroufel (2012). The differences of the electronic circuit in this paper from booth of them are in using power MOSFET complementary with $\mathrm{P}$ channel in positive line and $\mathrm{N}$ channel in ground line for three phase power module, and apply direct coupling mode between the PWM and the power modul.

\section{SYSTEM DESCRIPTION}

Figure 1 shows the basic circuit diagram of HEF 4752 from Philips with the three phase power module must be developed by the user. In this paper, the complete circuit bloc diagram showed at Figure 2. The low voltage three phase motor made from the conversion of one phase washing machine capacitor motor to three phase with single layer lap winding mode.

From Figure 1, in this paper, the potentio-meter for controlling the frequency of IC NE 566 (FCT; frequency control terminal) and potentiometer for controlling the voltage (VCT; voltage control terminal) via HEF 4047 both done by ATMEGA 32A microcontroller as seen in Fig. 2 with four step of action, motor stop, low speed, normal speed and high speed. To realize the action, the ohmic value of the two potentio divided into four resistor in the same value on each step. To avoid incompatibility of the operation voltage between the circuit and the microcontroller, magnetic relays are used in this circuit.

To drive the P-channel MOSFET power-module via CMOS IC 4050 in the positive side, there is a virtual ground 12 volt bellow the $\mathrm{V}_{\mathrm{CC}}$ and phase splitter to link the signal from the HEF 4752 to CMOS IC 4050 at both sides, upper and lower. This is done by low power MOSFET BS 107 . Three phase power source 32 volt $50 \mathrm{~Hz}$ supplied by three power transformer one phase 220 volt 5 ampere in star connection.

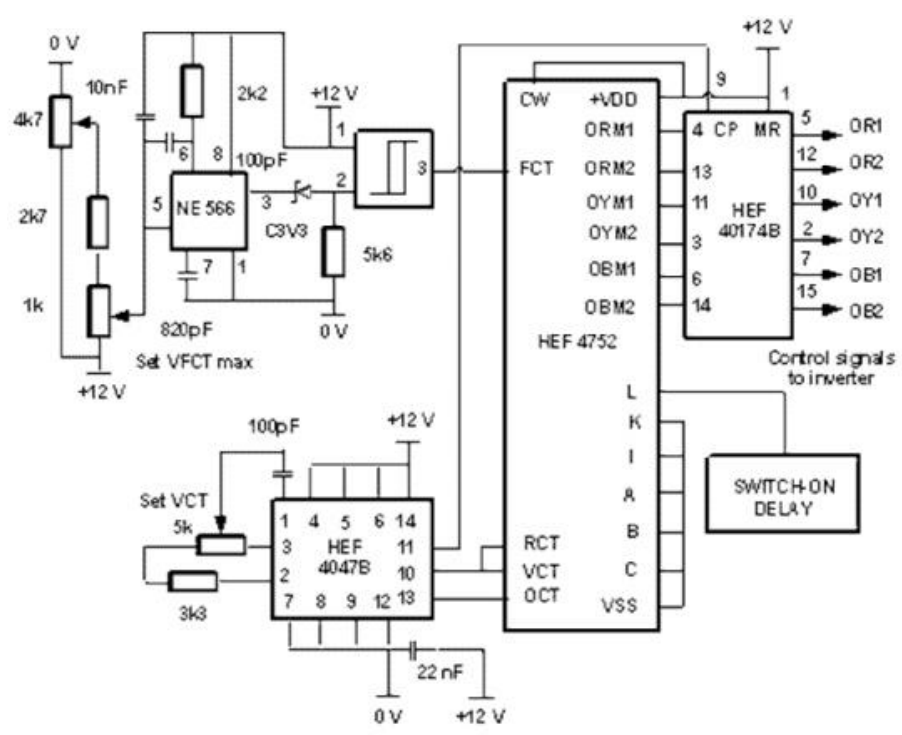

Fig. 1. Basic circuit of using HEF 4752

recommended by Philips semiconductors, 1995

\section{IMPLEMENTATION AND RESULT}

The block diagram in Fig.2 implemented in the physical modul showed in Fig 3. The motor laid in vertical position (the same as its function in washing machine), in the same casing with the whole circuit. 
The modul in Fig 3. has a trapezium form with dimension; $\mathrm{L}=59,5 \mathrm{~cm}, \mathrm{~W}=35 \mathrm{~cm}, \mathrm{H}=25 \mathrm{~cm}$ (rear), and $12 \mathrm{~cm}$ (front). Three phase led indicator for motor terminal voltage laid in the rear-left side, near the output terminals for measurement system. Three phase voltage input terminal laid in the rearright side near the led indicator for the input voltage. The push buttons (four) are used for motor operation ie: stop, low, medium, or high speed. The RPM and motor operation condition can be seen in the LCD screen. Fig. 4 shows the information in the LCD screen for starting motor operation, Fig 5 shows the statement after initial statement appear in Fig.4.

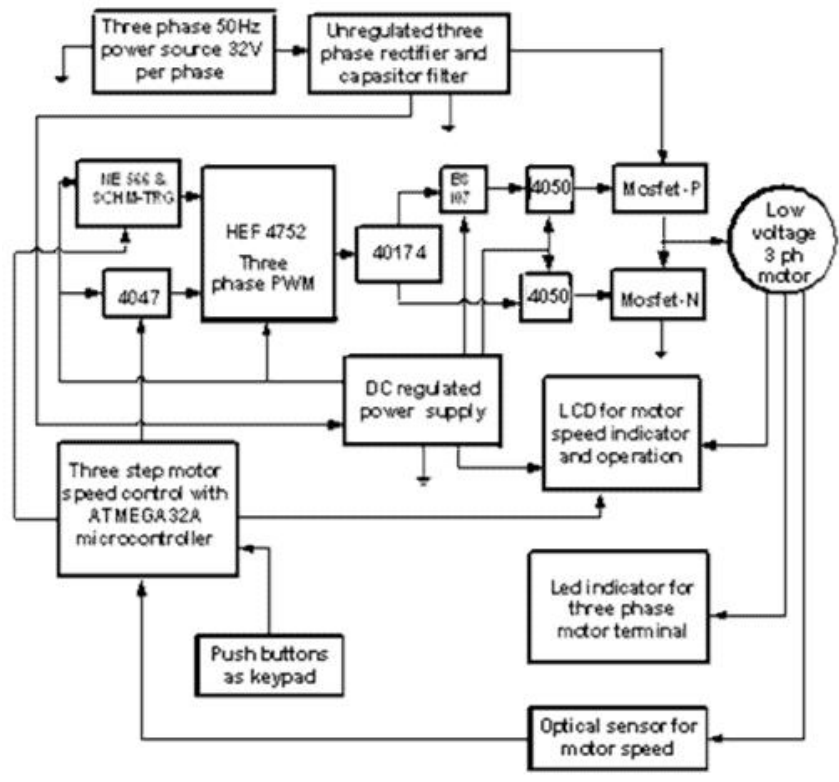

Fig. 2. Block diagram of circuit implementation for three phase motor speed control



Fig.3. The physical implementation of the block diagram in Fig.2

To start the motor, just press the push button on left side to make the frequency position active and then press push button on the upper side to increase the frequency or press the push button on the lower side to decrease the frequency. To make the voltage adjustment active, press the push button on right side to make and then press push button on the upper side to increase the voltage or press the push button on the lower side to decrease the voltage supply to the motor. Fig 6,7 and 8 show the result of the motor operation in low, medium and high speed condition, with the value of its speed.

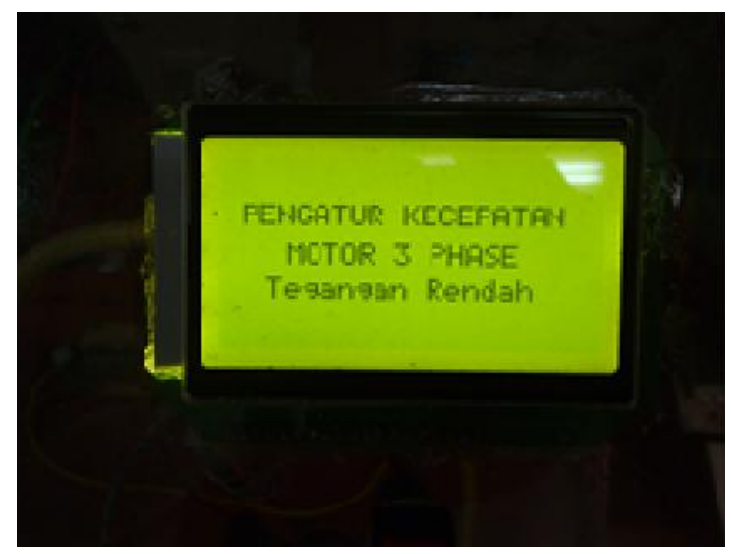

Fig.4 Initial statement on LCD screen after power input turned ON

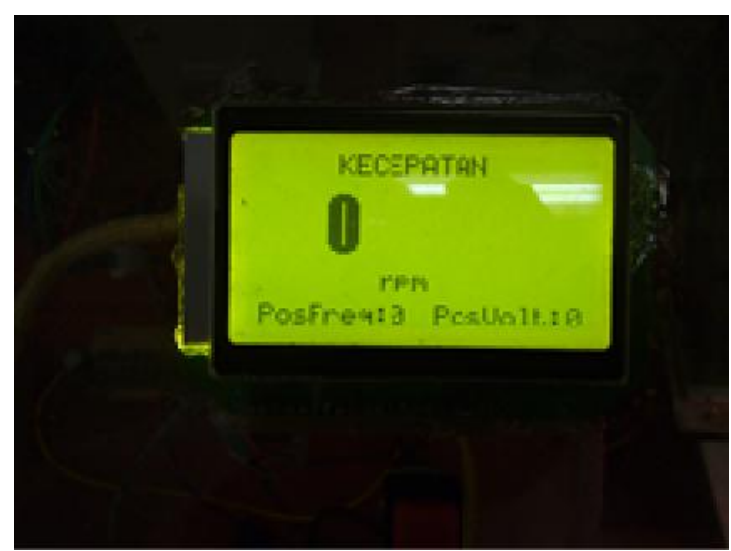

Fig 5. Starting to program the motor speed

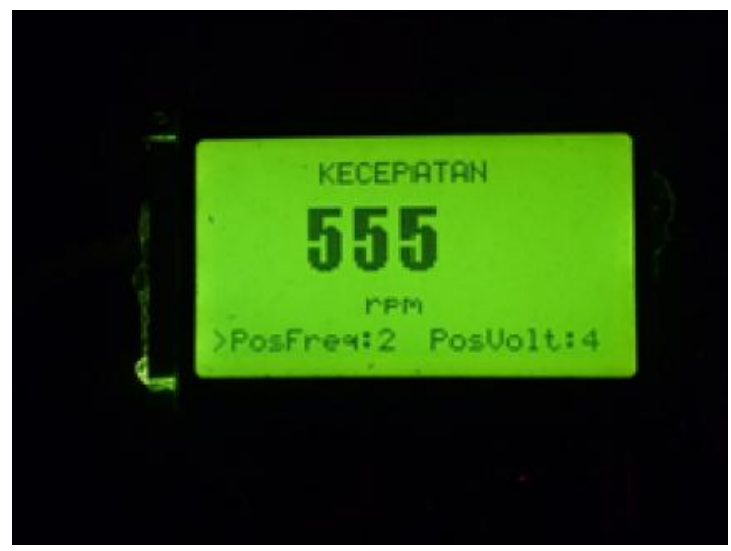

Fig. 6. Motor in low speed condition

Fig 9 shows the measurement of voltage shapes and its values. The voltages in the motor terminals measured with neutral point of the star connection. While motor in delta connection, the neutral point created via the point of the three 
resistor in star connection, named as 'virtual ground'. Fig10, motor in star connection.

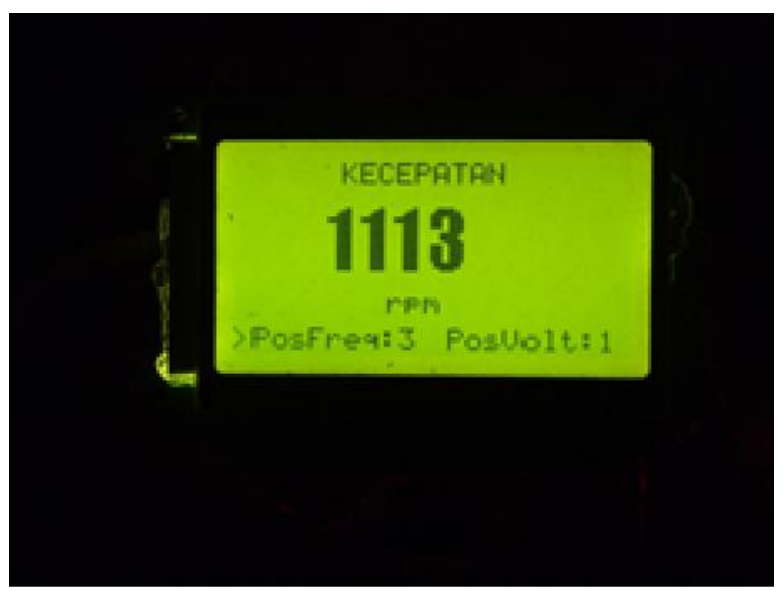

Fig. 7 Motor in medium speed condition

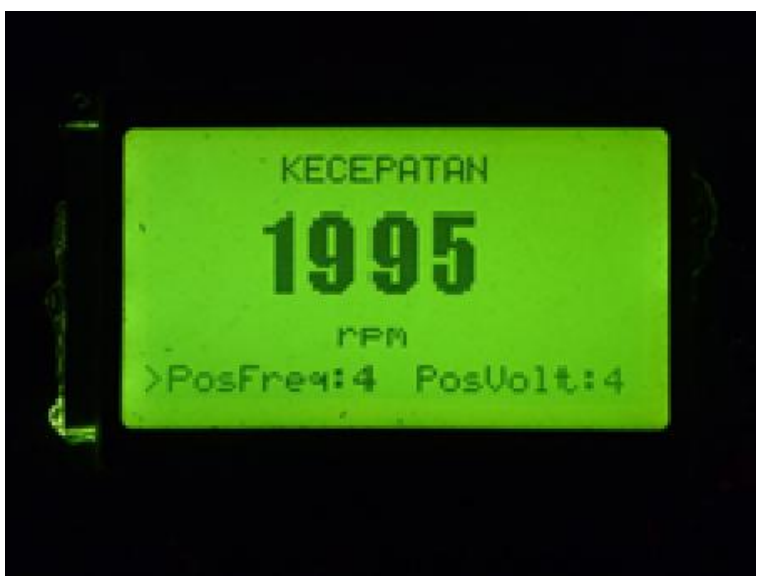

Fig 8. Motor in high speed condition.

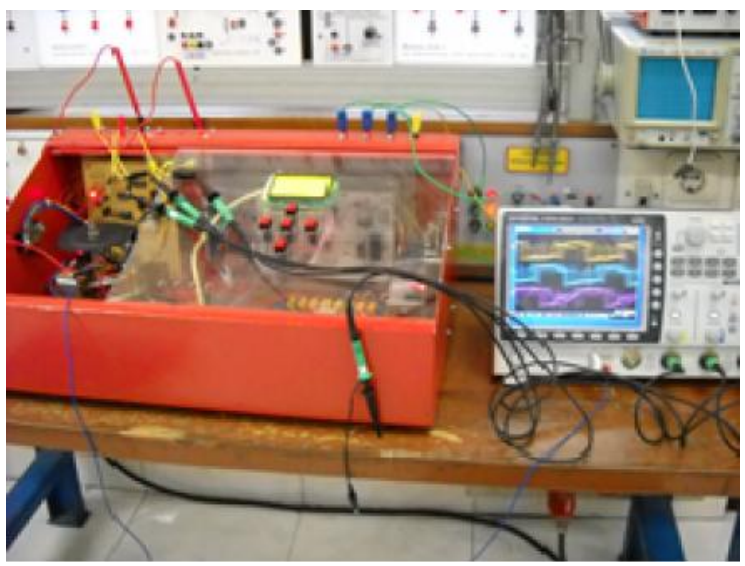

Fig.9 Taking data with motor in delta connection

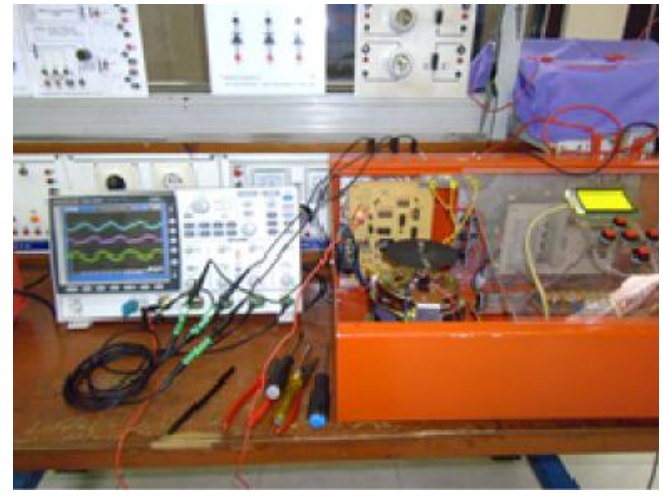

Fig.10 Taking data with motor in star connection

Fig 11 shows the motor terminal voltage in low speed; a) in star connection and b) in delta connection. Fig 12 and Fig 13 shows the motor terminal voltage in medium and high speed respectively; a) in star and b) in delta connection. The pictures show that in delta connection, the motor voltage loss of sinusoidal waves patern. In each mode of speed, there are a range of the value, this is a common fenomena in a digital measuring due to the pulses counting in each sampling.

Table 1 shows the complete data. In the setting of position 1 both for frequency and voltage level, there are no voltages in the output, so motor stop running. The differences between the output voltages per phase showed by analog and digital voltmeter measurement may caused by inner character of those meters how to react with spikes contained in the voltage being measured. Analog meter using SANWA YX 360 TRF with accuracy $\pm 4 \%$ in full scale, digital meter using SANWA CD800a with accuracy $\pm 1.6 \%+9$.

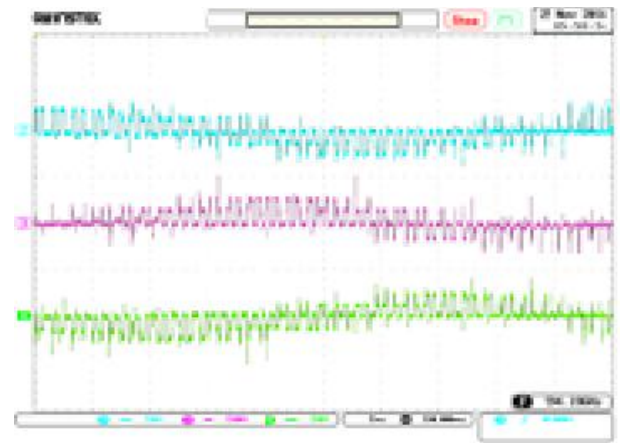

Fig 11a. Low speed, star connection 50V/div



Fig11b. low speed delta connection 20V/div 


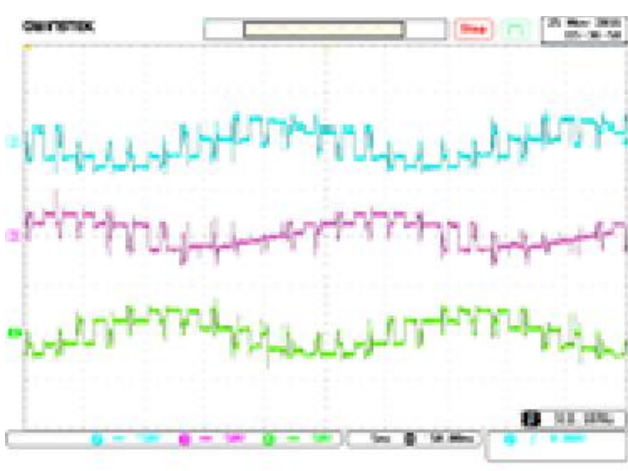

Fig 12a. Medium speed in star 50V/div

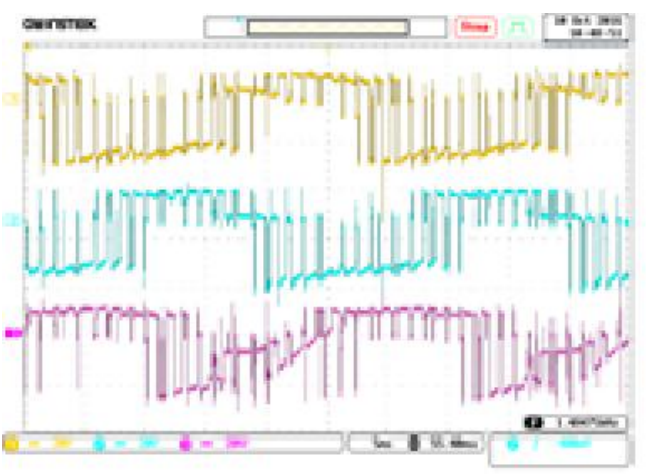

Fig 12b. Medium speed in delta (20V/div)

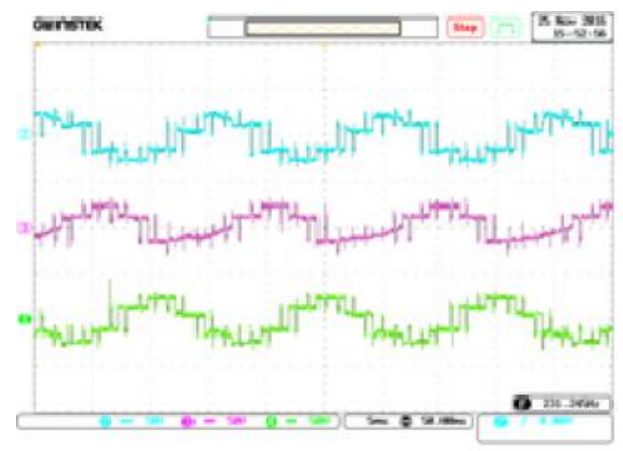

Fig 13a. High speed in star connection 50V/div

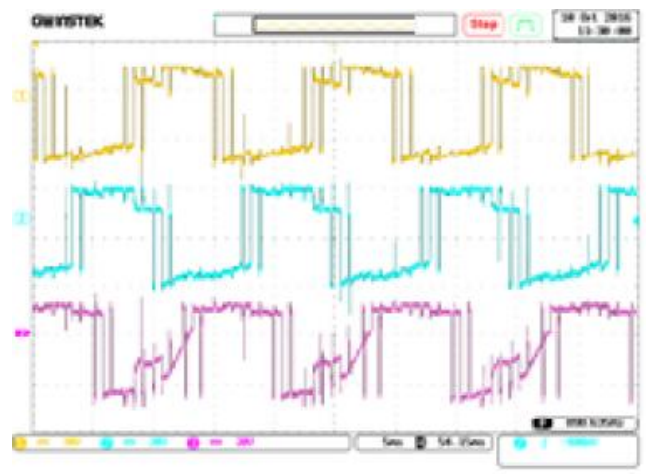

Fig 13b. High speed in delta connection 20V/div

Table 1. Motor speed, voltage and frequency

\begin{tabular}{|c|c|c|c|c|c|c|c|c|c|c|}
\hline & \multirow{2}{*}{\multicolumn{2}{|c|}{$\begin{array}{l}\text { Motor operation } \\
\text { via push buttons }\end{array}$}} & \multirow{4}{*}{$\begin{array}{c}\begin{array}{c}\text { Motor speed showed on } \\
\text { the LCD }\end{array} \\
(R P M)\end{array}$} & \multicolumn{6}{|c|}{$\begin{array}{c}\text { Voltage source: three phase } \\
32 \text { volt per phase } \\
\end{array}$} & \multirow{4}{*}{$\begin{array}{c}\begin{array}{c}\text { Frequency } \\
\text { (F=1/T from } \\
\text { oscilloscope } \\
\text { measurement) }\end{array} \\
\text { hertz }\end{array}$} \\
\hline & & & & & Pha & tagen & ement ( & & & \\
\hline & \multirow{2}{*}{$\begin{array}{l}\text { Freq } \\
\text { positon }\end{array}$} & \multirow{2}{*}{$\begin{array}{l}\text { Voltage } \\
\text { position }\end{array}$} & & \multicolumn{2}{|c|}{ Analog voltmeter } & \multicolumn{4}{|c|}{ Digital voltmeter } & \\
\hline & & & & $V R$ & $V S$ & $V T$ & $V R$ & $V S$ & $V T$ & \\
\hline 1 & 1 & 1 & 0 & 0 & 0 & 0 & 0 & 0 & 0 & 0 \\
\hline 2 & 2 & 1 & $537-573$ & 3,8 & 3,4 & 4,6 & 10,07 & 10,06 & 5,51 & 19,8 \\
\hline 3 & 3 & 1 & 1113-1176 & 8,2 & 7,95 & 9,5 & 15,03 & 14,97 & 11,38 & 38,46 \\
\hline 4 & 4 & 1 & $1995-2040$ & 10 & 10 & 11 & 17,87 & 17,70 & 15,17 & 67,79 \\
\hline 5 & 1 & 2 & 0 & 0 & 0 & 0 & 0 & 0 & 0 & \\
\hline 6 & 2 & 2 & $537-573$ & 4,2 & 4,2 & 4,0 & 10,21 & 10,21 & 5,64 & 19,8 \\
\hline 7 & 3 & 2 & $1122-1131$ & 8,2 & 8 & 9,7 & 15,4 & 15,00 & 11,16 & 38,46 \\
\hline 8 & 4 & 2 & $1995-2013$ & 10 & 10 & 11 & 17,98 & 17,70 & 15,15 & 67,79 \\
\hline 9 & 1 & 3 & Off & 0 & 0 & 0 & 0 & 0 & 0 & \\
\hline 10 & 2 & 3 & $555-573$ & 4,4 & 4,2 & 5,2 & 10,70 & 10,17 & 5,95 & 19,6 \\
\hline 11 & 3 & 3 & $1104-1140$ & 8,4 & 8,2 & 9,8 & 15,18 & 15,12 & 11,60 & 38,46 \\
\hline 12 & 4 & 3 & $1995-2004$ & 10 & 10 & 11 & 17,88 & 17,42 & 15,03 & 67,79 \\
\hline 13 & 1 & 4 & Off & 0 & 0 & 0 & 0 & 0 & 0 & \\
\hline 14 & 2 & 4 & $528-546$ & 4,6 & 4,4 & 5,6 & 10,96 & 11,02 & 6,24 & 19,6 \\
\hline 15 & 3 & 4 & 1104-1113 & 8,4 & 8,2 & 10 & 15,14 & 14,96 & 11,58 & 37,03 \\
\hline 16 & 4 & 4 & $1995-2022$ & 10 & 10 & 11 & 17,82 & 17,49 & 15,01 & 67,79 \\
\hline
\end{tabular}

\section{IV.CONCLUSION}

As the lab course, this modul must be used in motor star connection, seen from the patern of the motor terminal sinusoidal voltage. In order to make a smooth gradation of motor speed, the devider resistors booth in the FCT and VCT control via IC 566 and 4047 must be rearranged because of unlinear speed and voltage gradation to maintain constant volt per hertz for the motor.

The DC voltage input must be boosted after rectification to get more energy to the motor to make it runs smoothly in low speed mode. Thus the system need higher DC voltage to operate the motor in star connection 


\section{REFERENCES}

[1] Bellil Amer, Abdelkader Meroufel, 2012, "VSI

[2] PWM inverter feed induction machine using Volt per HertzControl Scheme", PRZEGLD ELEKTROTECHNICZNY (Electrical Review), ISSN 0033-2097, R.88 NR 5b/2012

[3] Patil M Pradep.,Sanjay L.Kurkute, 2006, "Speed control of three phase induction Motor using single phase supply along With active power factor correction", ACSE Journal, Volume (6), Issue (3)
[4] Philips Semiconductors, 1995, "HEF4752V LSI A.C. motor control circuit",

http://www.alldatasheet.com/datasheet.pdf/pdf/17778/PHILIPS/HEF475 2V.html [ Dec 20, 2012], 